\title{
Call Admission Control based on Bandwidth Allocation in Ultra Wide Band (UWB) Ad Hoc Networks
}

\author{
C. Viswanathan \\ Easwari Engineering College, \\ Chennai, India.
}

\author{
K. M. Mehata \\ Department of CSE, \\ B.S Abdur Rahman University, \\ Chennai, India
}

\author{
Latha Tamilselvan \\ Department of Information \\ Technology, \\ B. S. Abdur Rahman University \\ Chennai, India
}

\begin{abstract}
In Ultra-Wide Band (UWB) ad hoc networks, admission control and resource management are of major concern. The admission control algorithm helps in resolving infeasible power, reduces the congestion, and guarantees the quality of service (QoS) of unlicensed secondary users. The long acquisition time in the UWB transmissions and the near-sender blocking problems can be resolved by resource management effectively. In this paper, an admission control scheme and the resource management in UWB ad hoc routing is proposed. The bandwidth allocation and the bandwidth redistribution methods are employed here for the call admission control. Initially, bandwidth allocation is done using the HCF Controlled Channel Access (HCCA) centralized access which allows the $\mathrm{HC}$ to assign Transmission opportunity (TXOPs) to the ACs by taking into account the specific time constraints of each AC. After allocating the bandwidth, the free bandwidth is allocated to the other stations by estimating the traffic load. The call admission control is employed in order to guarantee the QoS. Simulation results show that the bandwidth allocation and call admission control scheme effectively solves the near-sender blocking problems in UWB ad hoc networks and improves achieved throughput and supports best effort traffic.
\end{abstract}

\section{Keywords}

Call admission control, Bandwidth allocation, Bandwidth redistribution, UWB ad-hoc networks.

\section{INTRODUCTION}

\subsection{Ultra Wide Band (UWB) Ad Hoc Networks}

The ULTRA-WIDEBAND system uses a short-duration burst of radio frequency energy for signal transmission and reception. The actual RF center frequency determination is quite difficult due to the extremely wide frequency band from the resultant waveforms [1]. The positional information having high precision can be recovered using UWB which helps in both the radar applications and the cellular systems applications. The telecommunication networks can be well organized by positioning data which helps in resource management and routing. Lower power levels can be rectified by using directivity in positioning [2].

A considerable degree of development can be seen in UWB systems which supports high data rates with low power consumption and low complexity in terms of transmission/reception operations since it is based on impulse radio (IR) technology. [3] Based upon the traffic types the management techniques are introduced individually. The guaranteed Quality of Service (QoS) also called best effort (BE) specifies the minimum degree of QoS throughout the connection which is used for the traffic type classification. [4]

The primary and active feature of radio networks have distributed architecture which helps in military applications. The infrastructure-less access in the ad hoc network and the coverage area which is faster and economically enhances the networking world providing vast applications in commercial sector. In areas where there is no communication support and where the infrastructure is hard to deploy, the wireless ad hoc networks effectively supports communication. In some places, the radio frequency spectrum doesn't have the permit for usage and thus the UWB applications are specifically highlighted in these regions.

Numerous possible routes can be discovered while transferring data from source to destination node. Due to this broadcasting nature of wireless media the neighboring UWB links also hamper in the data transfer. There is a difference in the received signal to noise ratio level which differentiates the higher quality and lower quality of routes in a specific time. So, the ad hoc networks can improve their performance by efficiently utilizing the possible radio resources by employing cooperative transmission strategies. [5]

\subsection{Routing in UWB Networks}

Without considering the preexisting infrastructure of the ad hoc networks, the wireless links can be established or disposed instinctively. Multi-hop Mobile Ad Hoc Network (MANET) are formed when the nodes operates as routers and the data packets are forwarded by cooperation of other nodes. The UWB technology is introduced as a promising transmission technology in the wireless communication networks. [6] Accurate information of the recovering distance can be provided by UWB [7].

The following issues are considered for the multi-hop routing in UWB.

Synchronization: A considerable synchronization overhead is caused due to uncoordinated MAC protocol.

Power: In order to meet the emission limits in UWB devices, the network performance can be optimized by managing the available power effectively.

Multi-User Interference (MUI): The power at the network level cannot be guaranteed for selecting optimized routes. 
Link reliability: Poor QoS is due to less reliability which can be reduced by incorporating the route selection procedure.

Traffic Load: Due to traffic load, one or more criteria can be frequently selected by a particular terminal.

End-to-end delay: QoS requirement is crucial in link reliability including ftp and http transfers. [8]

\subsection{Need for Admission Control in UWB Networks.}

The channel gain ratio in the admission control algorithm rejects the secondary users by provoking infeasible power control or low sum data rate. Resolving infeasible power control and improving sum data rate of an UWB cognitive network with less fairness loss are the major objective of admission control. The QoS of unlicensed secondary users need to be guaranteed by the power and admission control in the UWB cognitive radio networks without interfering with licensed primary users. [9]

Admission decision needs to be performed by a node for each request in the distributed power/rate allocation scheme. On admitting the request, local measurements of the system and control message exchanged helps in determining the transmission power and the rate. [10]

The network performance requirements can be met by admission control which eliminates the congestion. Due to unregulated access, the performance guarantees can be violated and thus the QoS aware networks require admission control. But the admission control algorithm is not required by the best effort networks. Like in cellular networks and centralized wireless networks, centralized schemes require the admission control schemes. [11]

The broadcast channel at each slot is heard initially by the sender during a call request arrival. The admission of a call can be determined by using this and the resources are allocated according to the power/rate levels for each sender. [12]

\subsection{Need for Resource Management in UWB Networks}

The long acquisition time in UWB transmissions can be rectified by resource management scheme which solves the near sender blocking problem. Resource management is required in order to consider the degradation of the achieved throughput which is caused due to long acquisition time. [12]

The IP pure "best effort" traffic like e-mail, WEB browsing, file transfer and data services having specific quality-of-service (QoS) requirements, are supported by radio resource sharing principles. [3]

In network access point (AP) of wireless LAN the resource management module is designed which is based on UWB as the fundamental physical transmission technique. Here AP is responsible for medium access control (MAC) functionalities which is helpful in transmission coordination and resource allocation of all devices. [13]

\subsection{Problem Identification and Proposed Solution}

In the previous works done, a cross-layer based routing protocol with a power saving technique for UWB ad hoc networks was presented. This protocol uses MAC and physical layer (PHY) specific information and maintains a separate route per Access Category (AC) for destination in its routing tables. The route selection is performed based on available bandwidth, contention delay, transmission delay and queuing delay at every link per AC from the available routes to a destination. In addition to this, we had also proposed an efficient power saving mechanism at the MAC layer which reduces power consumption on useless tasks, such as idle listening, collision, overhearing, and control overhead. The power saving technique uses an adaptive duty cycle mechanism based on the channel condition of a node.

Though this routing protocol provides reduced delay and energy consumption, there is a need for resource management and admission control in the routing technique. The admission control technique reduces the congestion, and guarantees the quality of service (QoS). The resource management in UWB networks is required to solve near-sender blocking problems, improves achieved throughput and supports best effort traffic. Thus as an extension to the routing protocol, a routing technique is implemented in UWB networks with admission control and resource management schemes.

\section{RELATED WORK}

Yi Shi, et al [1] have proposed Cross-Layer Optimization for Data Rate Utility Problem in UWB-Based Ad Hoc network. Here the maximizing capacity for a set of communication sessions is investigated in the form of data rate utility. A nonlinear programming problem is considered which accounts routing, scheduling, and power control. They had also developed a solution procedure based on the so-called branchand-bound framework.

Hongyu $\mathrm{Gu}$ et al [9] have considered the power and admission control for ultra-wideband (UWB) cognitive radio networks. This guarantees quality of service in secondary users without causing severe interference to primary users. Quasi-convex optimization problem is solved in this technique to maximize the minimal data rate of all admitted secondary users. An admission control algorithm is also proposed to reject secondary users, which induce infeasible power control or low sum data rate, by a new criterion - the channel gain ratio.

Hai Jiang et al [12] have proposed an effective resource management scheme for ultra-wideband (UWB) networks. Here simultaneous transmissions can be supported by inherent spread spectrum. In specific, they have presented a transmission frame structure tailoring to the UWB characteristics, and develop a novel control message exchange procedure.

Guerino Giancola et al [13] have presented the major results of our study on a resource manager model for an UWB network in the context of two application scenarios: (i) an UWB WLAN access network to a backbone and (ii) an UWB ad hoc network with peer-to-peer links. For both the above circumstances, The resource management scheme proposed here supports flows with different QoS requirements which involve physical parameters such as rate and power to specific traffic requirements.

F. Cuomo, et al [14] has proposed an approach based on the maximum extra interference. This interference can be tolerated by a QoS flow opposite to be applied in a distributed system where the optimal "minimum power approach" seems not applicable due to the high complexity required to reconfigure the transmission powers. 
Tommaso Melodia et al [15] have proposed a new cross-layer communication architecture based on the time-hopping impulse radio ultra wide band technology is described, whose objective is to reliably and flexibly deliver QoS to heterogeneous applications in WMSNs, by leveraging and controlling interactions among different layers of the protocol stack according to applications requirements.

\section{Call Admission Control Based on Bandwidth Allocation}

\subsection{Overview}

In this paper, a call admission control algorithm based on bandwidth allocation in UWB ad hoc networks is proposed The bandwidth allocation and the bandwidth redistribution methods are employed here for the call admission control. Initially, bandwidth allocation is done using the HCF Controlled Channel Access (HCCA) centralized access which allows the Hybrid Coordinator (HC) to assign Transmission opportunity (TXOPs) to the Access Categories (ACs) by taking into account the specific time constraints of each AC.

An UWB mobile Ad hoc network refers to a set of quality of service enabled nodes (QNs). Each QN has $N$ queues, with $N$ $=4$, one for any $\mathrm{AC}$ in the 802.11e proposal.

The queue length is estimated based on the time interval between two successive contention access points, average depletion rate and the disturbance. Then the transmission slots are assigned and the bandwidth is allocated accordingly. When the bandwidth allocated is more than the required bandwidth then the extra bandwidth is allocated to the other nodes. The extra bandwidth is calculated by estimating the traffic load. Thus the bandwidth redistribution helps in call admission control in the UWB ad hoc networks.

The bandwidth allocation in UWB networks solves the nearsender blocking problems, improves achieved throughput and supports best effort traffic.

\subsection{Queue Length Estimation}

Initially, the contention window $(\mathrm{CW})$ of a node is used to estimate the traffic load over a long period. For every $\mathrm{T}$ seconds, the $\mathrm{CW}$ of a node is calculated. The equation (1) determines the $\mathrm{CW}$ by exponentially weighing moving average method to the old contention window $\mathrm{CW}_{\text {old }}$ and sample contention window $\mathrm{CW}_{\text {sample }}[18]$.

$$
C W=\lambda * C W o l d+(1-\lambda) * C W \text { sample }
$$

We set $\lambda$ to 0.3 , which grants higher priority to the current sample $C W_{\text {sample }}$ [18].

The traffic load of this mobile node is calculated as the number of packets in the queue. More packets are passed through the interface queue when the traffic flows of the mobile node are higher. The below equation calculates the average queue length which gets updated every $\mathrm{T}$ seconds.

$$
Q=\beta^{*} Q(o)+(1-\beta) * Q(s)
$$

$\mathrm{Q}$ denotes the average queue length

$\mathrm{Q}(\mathrm{s})$ denotes the current queue length,

$\mathrm{Q}(\mathrm{o})$ denotes the old queue length,

$\beta$ is a constant and is set to 0.3 in our simulations [18].
The $\lambda$ and $\beta$ are the constants which have influence on the average value, the current traffic condition will impose. $\lambda$ and $\beta$ any number selected from the range $[0,1]$.

\subsection{Bandwidth Allocation}

Bandwidth allocation in UWB ad hoc networks can be provided by exploiting the HCF Controlled Channel Access (HCCA) centralized access method functionalities, which allows the HC to assign Transmission opportunity (TXOPs) to the ACs by taking into account the specific time constraints of each AC.

Let $I p$ be the time interval between two consecutive contention access points (CAPs). The bandwidth allocated to the node at every time interval Ip should be in a level such that it will drain each queue during next CAP. So the bandwidth assigned to drain the ith queue can be represented as the average depletion rate, Ai. The node is conscious of all the queue levels $\mathrm{Hi}$; $\mathrm{i}=1 \ldots \mathrm{T}$ at the beginning of each CAP where $T$ is the total number of traffic queues in the network.

\subsection{Transmission Opportunity (TXOP) Assignment}

In order to meet QoS constraints, HC allocates TXOPs to mobile nodes for every time interval Ip. Here the bandwidth $\mathrm{Ai}$ is transformed into a $\mathrm{TXOP}_{\mathrm{i}}$ assignment. This equation is specified if the ith queue is drained at data rate $\mathrm{Ri}$.

$$
\operatorname{TXOP}_{i}(s)=\frac{\left|A_{i}(s) \cdot I_{p}\right|}{R_{i}}+\eta
$$

Where TXOPi(s) is the TXOP assigned to the ith queue during the $s^{\text {th }}$ service interval

$\eta$ is the time overhead due to ACK packets, SIFS and PIFS time intervals.

The number of MSDUs corresponding to the amount of data $\left|A_{i}(s) \cdot I_{p}\right|$ to be transmitted determines the extra quota of TXOP which is due to the overhead $\eta$. On assuming all MSDUs with same nominal size which is sprécified into the TSPEC, it is possible to estimate the $\eta$ value. The queuing delay needs to be guaranteed if $\left|A_{i}(s) . I_{p}\right|$ does not correspond to a multiple of MSDUs. Here the TXOP assignment is rounded in excess which equals or lessens the target delay $\theta_{i}^{T}$.

[16]

\subsection{Call Admission Control}

In this section, Call Admission Control scheme is proposed to guarantee QoS. After the TXOP is allocated to the active traffic streams in each CAP, the following equation should be satisfied for admitting a new flow request.

$$
\frac{T_{X O P_{s+1}}}{I_{p}}+\sum_{i=1}^{s} \frac{T X O P_{i}}{I_{p}} \leq \frac{\rho-I p}{\rho}
$$

where

$\rho$ indicates the super frame duration

$I_{p}$ is the time used for HCCA traffic during the super frame.

Here the standard CAC test is modified by constant TXOPs by using a simple scheduler along with the bandwidth allocation algorithm. In addition to the sum of average source rates in TSPECs, the bandwidth is also taken into account for the CAC test. [16] 


\subsection{Bandwidth Redistribution}

After allocating the bandwidth, the remaining bandwidth in a station are redistributed to the other stations whenever the resources are not required locally for some time. The distribution of the free resources is calculated based upon the traffic load estimation.

In bandwidth redistribution, each node which has reserved resources, determines whether its communication load at discrete time intervals. If a sufficiently large amount of resources is observed to be temporarily free, the node will handover some of these resources to its "neighbors", by broadcasting a Extra Bandwith (EB) message.

Each node will continue to observe the arrival rate of its transmission queue. If its local load is increasing again, the node broadcasts a Bandwidth Recollection (RB) message to inform its neighbors that they are no longer allowed to make use of the extra bandwidth allocated.

For each stream, $\mathrm{HC}$ reassigns its resources on receiving the $E b$ and $R b$ messages. Here free bandwidth is added to the Contention Period, so it is fairly distributed since all back off entities can compete for access according to their priorities [17].

\subsection{Traffic Load Estimation}

The traffic load in UWB ad hoc networks are calculated based upon the contention window of a node.

The busy schedule of the medium is determined by BW and the contention and traffic situation of the node in ad hoc networks can be considered by $\mathrm{CW}$.

The channel cannot be accessed when the node has idle neighbors. The traffic load around the node can be determined by estimating the average contention of the channel around a mobile node

Based on the CW and queue length estimated in section 3.2, the local load of node i can be calculated as follow,

$$
\mathrm{W}=\mathrm{e}^{*} \mathrm{CW} / \mathrm{CW}_{\max }+(1-\mathrm{e}) * \mathrm{Q}(\mathrm{a}) / \mathrm{Q}(\mathrm{s})
$$

The $\mathrm{Q}$ (a) means the low load. The higher value of the $\mathrm{W}$ means the larger load. The selection of constant $e$ is to balance the effects of the two factors $\mathrm{CW}$ and $\mathrm{Q}$. In our simulation we set $\mathrm{e}$ to 0.5 , which grants the same priority to the two factors. [18]

There are two thresholds $\mathrm{W}_{\min }$ and Wmax which indicate the minimum and maximum threshold values for $\mathrm{W}$, respectively.

Let $\mathrm{W}\left(\mathrm{t}_{\mathrm{i}}\right)$ denote the local load $\mathrm{W}$ at time interval $\mathrm{t}_{\mathrm{i}}$.

If $\mathrm{W}\left(\mathrm{t}_{\mathrm{j}}\right)<\mathrm{Wmin}$, then

EB message can be broadcasted to allocate the free bandwidth to the neighbors.

Else if $W\left(t_{i}\right)>W m a x$, then

RB message can be broadcasted to withdraw the allocated free bandwidth from the neighbors.

The time interval $t_{i}$ can be incremented as

$$
\mathrm{t}_{\mathrm{i}}=\mathrm{t}_{\mathrm{i}}+\mathrm{dt}
$$

where dt represents the small increment for the time interval.

\section{PERFORMANCE EVALUATION}

\subsection{Simulation Model and Parameters}

NS2 [19] is used to simulate our proposed algorithm. The NS2 UWB MAC and PHY simulator patch is applied for the 2.29 version of standard ns- 2 . This code is a modification and extension of ns-2 to support impulse radio UWB wireless networking. The two main components that are added to the plain ns-2 is the DCC-MAC layer (mac-ifcontrol. $\{\mathrm{cc}, \mathrm{h}\}$ ) and a model of an impulse radio UWB physical layer (interferencephy. $\{\mathrm{cc}, \mathrm{h}\})$ assuming time-hopping, pulse position modulation and convolutional code for channel coding.

In this simulation, the IFControl MAC protocol with CodedPPM modulation and Tarokh propagation model is used.

In this simulation, the flows are varied as $1,2,3,4$ and 5 . The mobile nodes move in a 1250 meter x 1250 meter square region for 10 seconds simulation time. It is assumed that each node moves independently with the same average speed. All nodes have the same transmission range of 250 meters. In our simulation, the speed is $10 \mathrm{~m} / \mathrm{s}$.

The simulation settings and parameters are summarized in table.

Table I: Simulation parameters

\begin{tabular}{|l|l|}
\hline No. of Nodes & 50 \\
\hline MAC protocol & MAC-IFControl \\
\hline Propagation Model & Tarokh \\
\hline Modulation & CodedPPM \\
\hline Area Size & 1250 X 1250 \\
\hline Mac & 802.11 \\
\hline Radio Range & $250 \mathrm{~m}$ \\
\hline Simulation Time & $10 \mathrm{sec}$ \\
\hline Traffic Source & CBR and Video \\
\hline Packet Size & 512 \\
\hline Mobility Model & Random Way Point \\
\hline Speed & $10 \mathrm{~m} / \mathrm{s}$ \\
\hline Pause time & $5 \mathrm{sec}$ \\
\hline No. of Flows & $1,2,3,4$ and 5 \\
\hline Rate & $250,500,750$ and $1000 \mathrm{~Kb}$. \\
\hline
\end{tabular}

\subsection{Performance Metrics}

The performance of the proposed CACBA scheme is compared with the existing Effective Resource Management (ERM) [12] scheme according to the following metrics.

Average end-to-end delay: The end-to-end-delay is averaged over all surviving data packets from the sources to the destinations.

Drop: It is the number of packets dropped during the transmission.

Bandwidth: It is the measure of the total received bandwidth.

Delivery Ratio: It is the ratio between number of packets received and the number of packets sent.

\section{A. Based on Flows}

In this case, the number of flows is varied for both CBR and VBR traffic flows from 1 to 5 , keeping the traffic rate as $250 \mathrm{~Kb}$. 


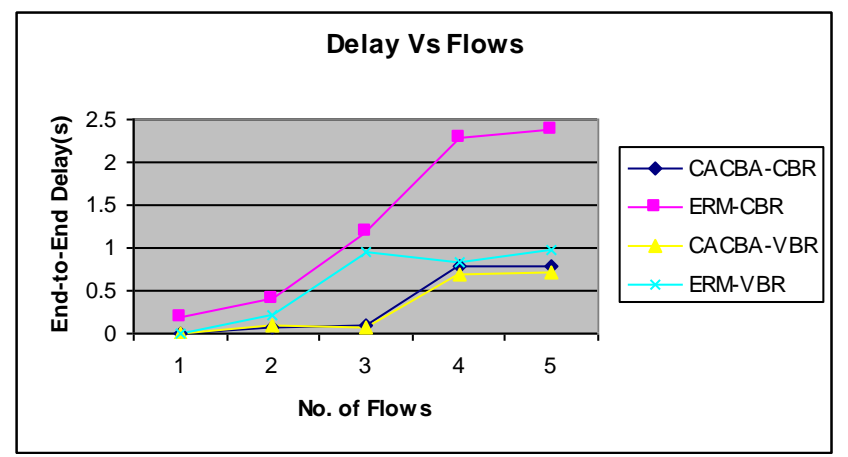

Fig 1: Flows Vs Delay

Figure 1 gives the delay occurred for both the techniques when the number of traffic flows is increased. When the number of flows of both CBR and VBR are varied from 1 to 5, there will be severe contention and hence more packets has to wait in the queue. As it can be seen from the figure, the delay is high in case of CBR traffic when compared to VBR traffic. But CACBA has $69 \%$ less delay when compared to ERM for the CBR traffic. In case of VBR traffic, CACBA has $42 \%$ lesser delay than ERM.

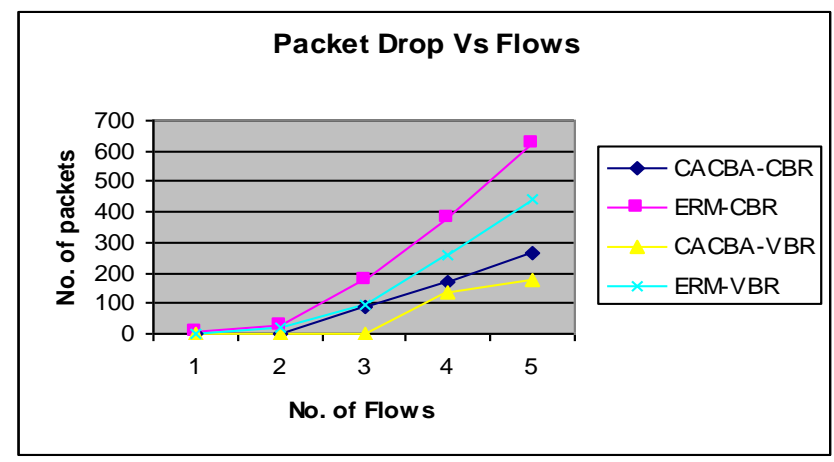

Fig 2: Flows Vs Packet Drop

Figure 2 shows the number of packets dropped using both the techniques when the number of traffic flows is increased. When the number of flows of both CBR and VBR are varied from 1 to 5 , there will be a queue overflows resulting in more packet drops. As it can be seen from the figure, the CBR traffic has more packet drops when compared to VBR traffic for both CACBA and ERM since at high contention, the packets of lower priority CBR traffic are dropped. However CACBA has $69 \%$ less drops when compared to ERM for the CBR traffic. In case of VBR traffic, CACBA has $68 \%$ lesser drops than ERM.

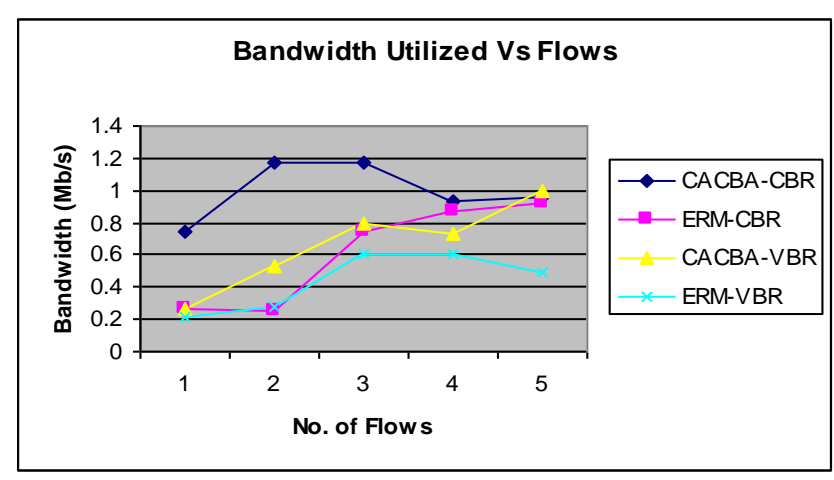

Fig 3: Flows Vs Bandwidth
Figure 3 shows the received bandwidth using both the techniques when the number of traffic flows is increased. When the number of flows of both CBR and VBR are varied from 1 to 5 , there will be a queue overflow so that additional bandwidth cannot be allocated for VBR flows. Hence it can be seen from the figure, the CBR traffic has higher received bandwidth when compared to VBR traffic for both CACBA and ERM. It can be observed that CACBA received $38 \%$ more bandwidth when compared to ERM for the CBR traffic. In case of VBR traffic, CACBA received 32\% higher bandwidth than ERM.

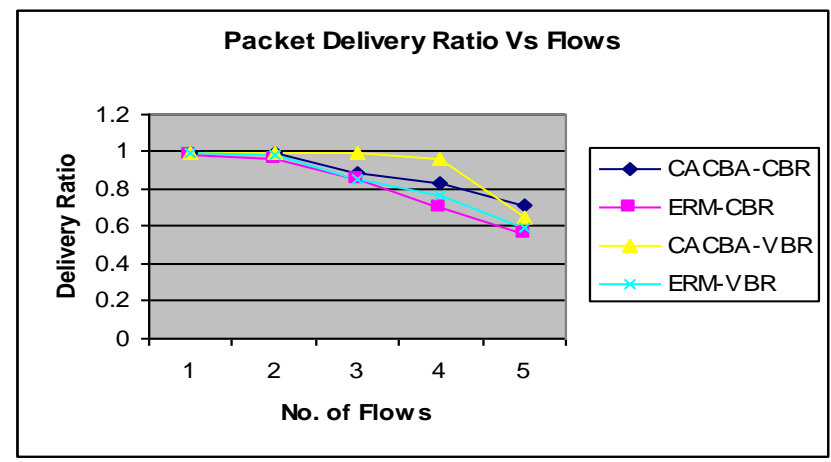

Fig 4: Flows Vs Delivery Ratio

Figure 4 shows the packet delivery ratio using both the techniques when the number of traffic flows is increased. When the number of flows of both CBR and VBR are varied from 1 to 5 , there will be queue overflows resulting in degraded packet delivery ratio. Figure 4 shows that CBR traffic has less packet delivery ratio when compared to VBR traffic for both CACBA and ERM since CBR traffic flows has more packet drops at high contention as per figure 2. Also CACBA has 9\% more packet delivery ratio when compared to ERM for the CBR traffic. In case of VBR traffic, CACBA has $6 \%$ higher delivery ratio, than ERM.

\section{B. Based on Rate}

In this case, the traffic rate is varied as $250,500,750$ and $1000 \mathrm{~Kb}$ for both CBR and VBR traffic with 5 flows.

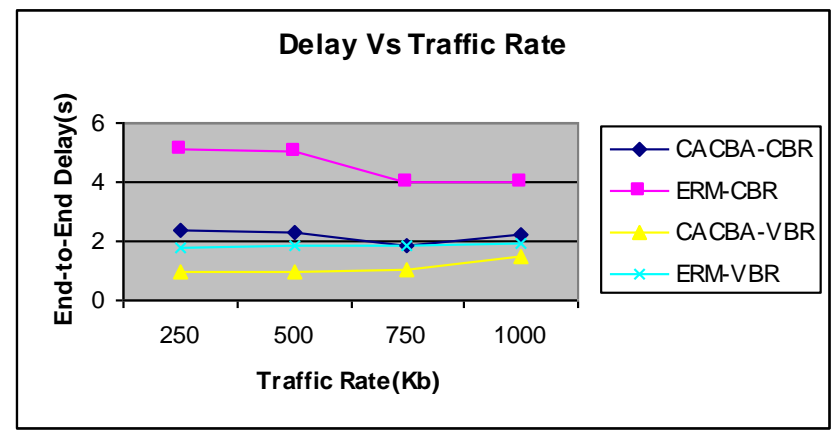

Fig 5: Rate Vs Delay

Figure 5 gives the delay occurred for both the techniques when the data rate is increased. When the data rate of both CBR and VBR are varied from $250 \mathrm{~Kb}$ to $1000 \mathrm{~Kb}$, the required bandwidth for CBR flows will be increasing hence more CBR packets has to wait in the queue. As it can be seen from the figure, the delay is high in case of CBR traffic when compared to VBR traffic. But CACBA has $62 \%$ less delay when compared to ERM for the CBR traffic. In case of VBR traffic, CACBA has $40 \%$ lesser delay than ERM. 


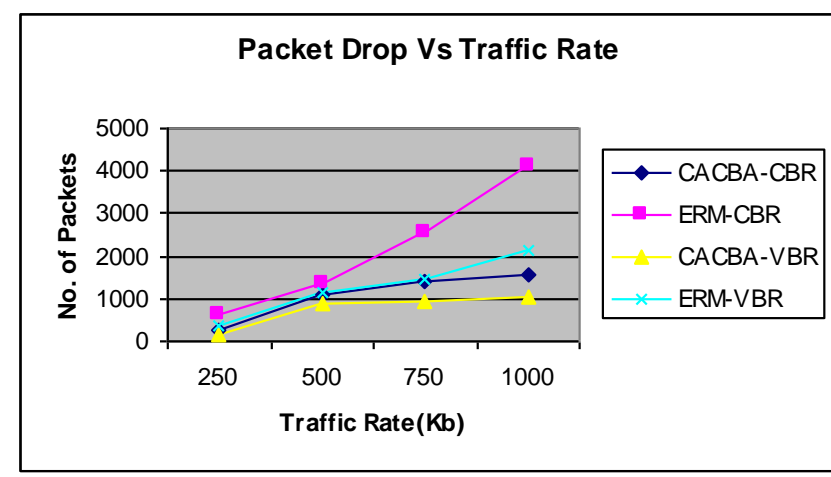

Fig 6: Rate Vs Drop

Figure 6 shows the number of packets dropped using both the techniques when the traffic rate is increased. When the rate of both CBR and VBR traffic are varied from $250 \mathrm{~Kb}$ to $1000 \mathrm{~Kb}$, there will be queue overflows resulting in more packet drops. As it can be seen from the figure, the CBR traffic has more packet drops when compared to VBR traffic for both CACBA and ERM since at high contention, the packets of lower priority CBR traffic are dropped. However CACBA has $45 \%$ less drops when compared to ERM for the CBR traffic. In case of VBR traffic, CACBA has $40 \%$ lesser drops than ERM.

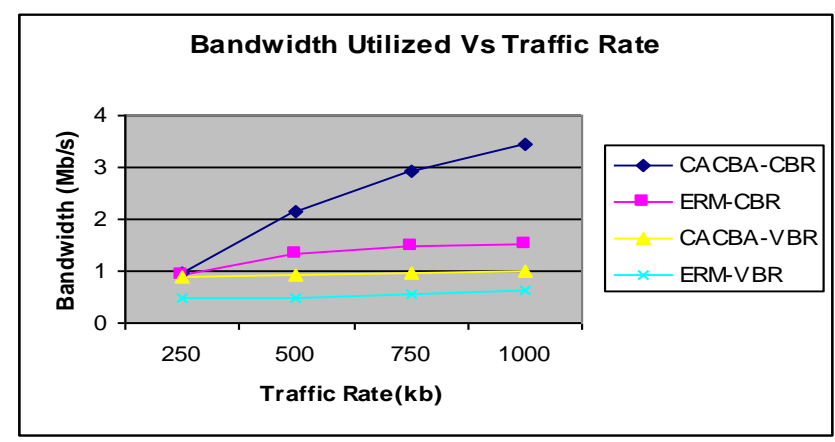

Fig 7: Rate Vs Bandwidth

Figure 7 shows the received bandwidth using both the techniques when the traffic rate is increased. When the rate of both CBR and VBR traffic are varied, there will be a queue overflow so that additional bandwidth cannot be allocated for VBR flows. Hence it can be seen from the figure, the CBR traffic has higher received bandwidth when compared to VBR traffic for both CACBA and ERM. It can be observed that CACBA received $51 \%$ more bandwidth when compared to ERM for the CBR traffic. In case of VBR traffic, CACBA received $42 \%$ higher bandwidth than ERM.

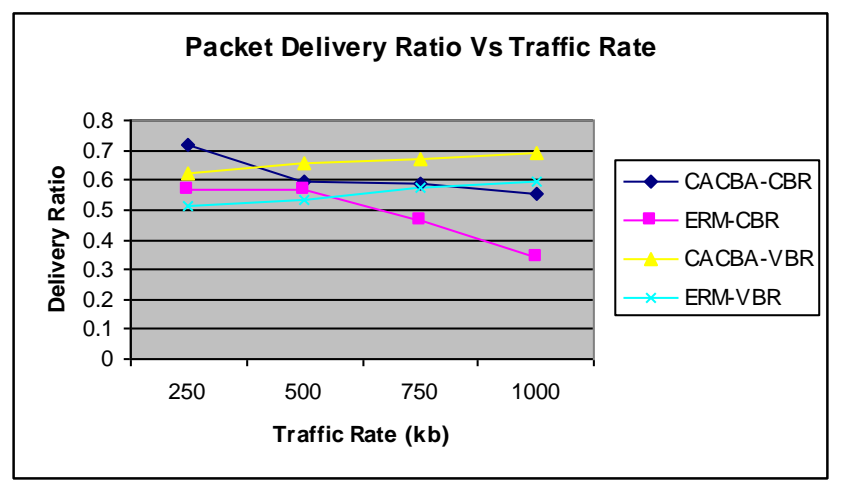

Fig 8: Rate Vs Packet Delivery Ratio
Figure 8 shows the packet delivery ratio using both the techniques when the traffic rate of flows is increased. When the rate of both CBR and VBR traffic are varied, there will be queue overflows resulting in degraded packet delivery ratio. Figure 8 shows that $\mathrm{CBR}$ traffic has less packet delivery ratio when compared to VBR traffic for both CACBA and ERM, since CBR traffic flows has more packet drops at high contention as per figure 6 . Also CACBA has $21 \%$ more packet delivery ratio when compared to ERM for the CBR traffic. In case of VBR traffic, CACBA has $16 \%$ higher delivery ratio, than ERM.

From the performance analysis, we can say that CACBA performs better for CBR traffic, than VBR traffic, in terms of percentage of improvement.

\section{CONCLUSION}

In this paper, an admission control scheme and the resource management in UWB ad hoc routing is proposed. The bandwidth allocation and the bandwidth redistribution methods are employed here for the call admission control. Initially, bandwidth allocation is done using the HCF Controlled Channel Access (HCCA) centralized access which allows the HC to assign Transmission opportunity (TXOPs) to the ACs by taking into account the specific time constraints of each AC. The queue length is estimated based on the time interval between two successive contention access points, average depletion rate and the disturbance. Z-transformation is used to determine steady state queuing delay. Then the transmission slots are assigned and the bandwidth is allocated accordingly. When the bandwidth allocated is more than the required bandwidth then the extra bandwidth is allocated to the other stations. The extra bandwidth is calculated by estimating the traffic load. The call admission control is employed in order to guarantee the QoS. From the simulation results, it has been shown that the bandwidth allocation and call admission control scheme effectively solves the near-sender blocking problems in UWB ad hoc networks and improves achieved throughput and supports best effort traffic.

\section{REFERENCES}

[1] Yi Shi, Y. Thomas Hou, and Hanif D. Sherali "CrossLayer Optimization for Data Rate Utility Problem in UWB-Based Ad Hoc Networks" IEEE TRANSACTIONS ON MOBILE COMPUTING, VOL. 7, NO. 6, JUNE 2008.

[2] Luca De Nardis, Pierre Baldi, and Maria Gabriella Di Benedetto "UWB Ad hoc networks" 2002 IEEE Conference on Wideand systems and technolgies.

[3] Francesca Cuomo, Cristina Martello, Andrea Baiocchi and Fabrizio Capriotti "Radio Resource Sharing for Ad Hoc Networking With UWB" IEEE JOURNAL ON SELECTED AREAS IN COMMUNICATIONS, VOL. 20, NO. 9, DECEMBER 2002.

[4] Tomaso Erseghe, Nicola Laurenti, Pietro Nicoletti, and Andrea Sivieri "An Algorithm for Radio Resource Management in UWB Ad Hoc Networks with Concurrent Guaranteed QoS and Best Effort Traffic" Wireless Personal Multimedia Communication 2004.

[5] Shouhong Zhu and Kin K. Leung "Distributed Cooperative Routing for UWB Ad-Hoc Networks" IEEE Communications Society subject matter experts for publication in the ICC 2007 proceedings. 
[6] Samer Bali and Prof. Dr.-Ing. Klaus Jobmann "ROUTING PROTOCOLS FOR ULTRA-WIDEBAND MOBILE AD HOC NETWORKS” 2008.

[7] Luca De Nardis, Guerino Giancola, and Maria-Gabriella Di Benedetto Power-aware design of MAC and routing for UWB networks" CAMAD'04 Workshop, IEEE Global Communications Conference 2004,

[8] Maria-Gabriella Di Benedetto and Luca De Nardis "Cognitive routing in UWB networks" IEEE International Conference on UWB 2006 (ICUWB2006),

[9] Hongyu Gu and Chenyang Yang "Power and Admission Control for UWB Cognitive Radio Networks" IEEE Communications Society subject matter experts for publication in the ICC 2008 proceedings.

[10] Xuemin (Sherman) Shen, Weihua Zhuang, Hai Jiang, and Jun Cai "Medium Access Control in Ultra-Wideband Wireless Networks" IEEE TRANSACTIONS ON VEHICULAR TECHNOLOGY, VOL. 54, NO. 5, SEPTEMBER 2005.

[11] Luca De Nardis, and Maria-Gabriella Di Benedetto "Medium Access Control design for UWB Communication Systems: review and trends" JOURNAL OF COMMUNICATIONS AND NETWORKS, VOL. 5, NO. 3, SEPTEMBER 2003

[12] Hai Jiang, Kuang-Hao Liu, Weihua Zhuang, and Xuemin (Sherman) Shen "An Effective Resource Management Scheme for UWB Networks With Simultaneous Transmissions" IEEE TRANSACTIONS ON WIRELESS COMMUNICATIONS, VOL. 6, NO. 6, JUNE 2007.
[13] Guerino Giancola, Cristina Martello, Francesca Cuomo and Maria-Gabriella Di Benedetto "Radio resource management in infrastructure-based and ad hoc UWB networks" WIRELESS COMMUNICATIONS AND MOBILE COMPUTING Wirel. Commun. Mob. Comput. 2005; 5:581-597.

[14] F. Cuomo, C. Martello, and S. Caputo "An interferencecontrolled admission control scheme for QoS support in distributed UWB networks" Proceedings of IST mobile 2003.

[15] Tommaso Melodia, and Ian F. Akyildiz "Cross-Layer QoS-Aware Communication for Ultra Wide Band Wireless Multimedia Sensor Networks" IEEE JOURNAL ON SELECTED AREAS IN COMMUNICATIONS, VOL. 28 , NO. 5, JUNE 2010.

[16] G. Boggia, P. Camarda, L. A. Grieco, and S. Mascolo "Feedback Based Bandwidth Allocation with Call Admission Control for Providing Delay Guarantees in IEEE 802.11e Networks" IEEE ACM transactions April 2007.

[17] J"urgen Wolf Stephan Heckm"uller and Bernd E. Wolfinger "Dynamic Resource Reservation and QoS Management in IEEE 802.11e Networks" 2005

[18] Xuemei Gao, Xinming Zhang, Dong Shi, Fengfu Zou, and Wenbo Zhu "Contention and Queue-aware Routing Protocol for Mobile Ad h oc Networks" IEEE conference Wireless Communications, Networking and Mobile Computing, 2007. WiCom 2007. International Conference

[19] Network Simulator: http:///www.isi.edu.ns/nam. 